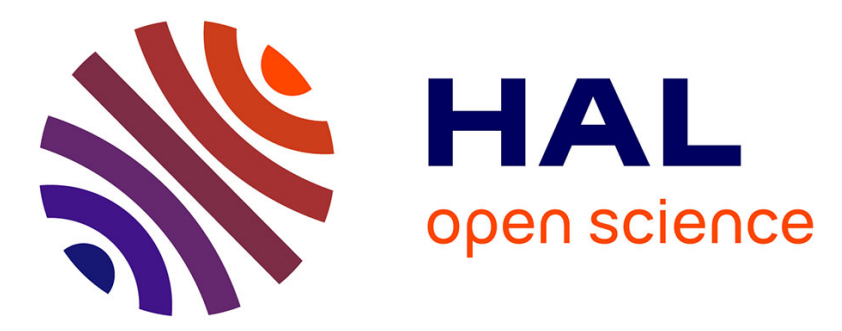

\title{
Opportunistic and Efficient Resource Block Allocation Algorithms for LTE Uplink Networks
}

Fatima Zohra Kaddour, Mylène Pischella, Philippe Martins, Emmanuelle Vivier, Lina Mroueh

\section{- To cite this version:}

Fatima Zohra Kaddour, Mylène Pischella, Philippe Martins, Emmanuelle Vivier, Lina Mroueh. Opportunistic and Efficient Resource Block Allocation Algorithms for LTE Uplink Networks. WCNC 2013, Apr 2013, Shanghai, China. pp.1-5. hal-00822522

\section{HAL Id: hal-00822522 \\ https://hal.science/hal-00822522}

Submitted on 14 Oct 2014

HAL is a multi-disciplinary open access archive for the deposit and dissemination of scientific research documents, whether they are published or not. The documents may come from teaching and research institutions in France or abroad, or from public or private research centers.
L'archive ouverte pluridisciplinaire HAL, est destinée au dépôt et à la diffusion de documents scientifiques de niveau recherche, publiés ou non, émanant des établissements d'enseignement et de recherche français ou étrangers, des laboratoires publics ou privés. 


\title{
Opportunistic and Efficient Resource Block Allocation Algorithms for LTE Uplink Networks
}

\author{
Fatima Zohra Kaddour ${ }^{1,2}$, Mylene Pischella ${ }^{3}$, Philippe Martins ${ }^{1}$, Emmanuelle Vivier ${ }^{2}$, Lina Mroueh ${ }^{2}$ \\ ${ }^{1}$ Telecom ParisTech, 46 rue Barrault, Paris, France \\ 2 ISEP, 28 rue N-D des Champs, Paris, France \\ ${ }^{3}$ CNAM, 292 rue Saint-Martin, Paris, France
}

\begin{abstract}
This work proposes two new Resource Block (RB) allocation algorithms for the LTE uplink. They take into account the RB adjacency constraint and update the allocation metric. Two different heuristics are proposed: an Opportunistic and Efficient RB allocation Algorithm (OEA) and a Quality of Service based Opportunistic and Efficient RB allocation Algorithm (QoS based OEA). Both algorithms seek to maximize the aggregate throughput and avoid RB wastage unlike other algorithms in the literature. The complexity of the proposed algorithms are also computed analytically and compared to other well known heuristics.
\end{abstract}

Keywords: Resource Block allocation, SC-FDMA, LTE.

\section{INTRODUCTION}

Single Carrier Frequency Division Multiple Access (SCFDMA) was adopted for the 3GPP Long Term Evolution (LTE) uplink networks. In addition to the Orthogonal Frequency Division Multiple Access (OFDMA) technique advantages, the SC-FDMA allows a low Peak to Average Power Ratio (PAPR) by considering the whole allocated Resource Block (RB) as a single carrier and shares the transmission power equally over the allocated subcarriers. A low PAPR decreases the mobile battery consumption, the equalizer complexity and the terminal cost. Packet scheduling in LTE occurs every subframe, where a scheduling period of one subframe is alternatively known as Transmission Time Interval (TTI) with $1 \mathrm{~ms}$ duration. In fact, the scheduling algorithm shall be simple and efficient. Due to the multi-user diversity gain, channel dependent scheduling is performed (i.e. using the channel conditions as a metric). According to this chosen metric, the packet scheduler applies its allocation policy to determine the RBs allocated to the user that maximizes the satisfaction level of the system, such as the aggregate throughput. The allocation policy used by the scheduler in the uplink must consider: (i) the Modulation and Coding Scheme (MCS) robustness: defined by using the same MCS over the whole RBs allocated to one user equipment (UE) and (ii) the contiguity constraint required by the SC-FDMA technique: one UE must be allocated adjacent RBs. Due to these two constraints, most RB allocation algorithms proposed in the literature for the downlink cannot be directly applied to the uplink. The RB allocation problem in LTE uplink systems has been addressed in many papers in the literature. The optimal RB allocation method can be given using the Binary Integer Programming (BIP) as proposed in [1], where the users' transmission power is set to its maximum and the throughput computation is done without considering the MCS robustness. The same optimal method BIP is proposed in [2] where the objective function is to minimize each individual user transmission power. The BIP is solved using the branch and bound method which has an NP-hard complexity. Then, the authors of [3] [4] propose to use the Hungarian algorithm, which is less complex, to find the optimal mapping between UE's and RBs. The Hungarian algorithm was adapted to the SC-FDMA constraints by grouping the contiguous RBs in equal Resource Chunks (RC). The authors of [4] propose to add a fairness factor in the metric computation to enable a fair RC allocation among the users. In [5] the authors propose a Heuristic Localized Gradient Algorithm (HLGA) which searches the pairs (RB-UE) that maximize the metric first, and allocates them to the concerned users, one pair after the other. If RBs assigned to the selected user are not adjacent, this user is also assigned the in-between RBs. When all users are served, the remaining free RBs are assigned to users that satisfy the contiguity constraint. The Frequency Domain Packet Scheduling - Largest Metric value First (FDPS-LMF) proposed in [6] is similar to the one used in HLGA, but the management of the remaining free RBs is not specified. In [7] Recursive Maximum Expansion (RME) scheduler is proposed. This scheduler recursively searches the pair (RB-UE) which maximizes the metric and expands the RBs allocation on the two sides of the selected RB as long as the considered UE maximizes the metric. RME proposes the same remaining free RBs management as HLGA. The allocation policies of these algorithms are opportunistic, but as they use the proportional fair metric studied in [8], which is determined by the ratio between the logarithm of the instantaneous user throughput and the average throughput, they become more fair.

In this paper, we propose a new Opportunistic and Efficient $\mathrm{RB}$ allocation Algorithm (OEA). It seeks to maximize the aggregate throughput, but the RB allocation is efficient because the RBs are allocated to a UE only if its throughput is improved. In order to prevent the wastage of RBs (i.e. to make sure that new RBs will not be allocated to an user if it leads to a zero throughput), we update the metric of the concerned UE in the entire bandwidth before each RB allocation validation. Once the RB allocation is performed, the Physical Downlink Control Channel $(\mathrm{PDCCH})$, with the standard procedure, is used to inform each mobile about the RBs that are allocated to it [9]. A second variant of Quality of Service based OEA 
(QoS based OEA) is also presented.

The paper is organized as follows. In Section II we define the system model and parameters used for simulations. Section III describes the proposed heuristics: OEA and QoS based OEA. Section IV evaluates the computational complexity of the discussed algorithms. In Section V the algorithms performances comparison is presented. Finally Section VI concludes the paper.

\section{SySTEM MODEL}

We consider a LTE Network composed of 19 hexagonal cells. Each cell is provided with tri-sectored Base Station (BS). To mitigate Inter-Cell Interference (ICI), we allocate different bandwidths $B$ to each sector and a $1 \times 3 \times 1$ frequency reuse pattern. The smallest radio resource that can be allocated to a user is one RB which is described by $N_{s c}^{R B}$ adjacent subcarriers and $N_{s y m b}^{U L}$ SC-FDMA symbols, where $N_{s c}^{R B}=12$ and $N_{s y m b}^{U L}$ is equal to 6 or 7 according to the Cyclic Prefix (CP) configuration [10]. We suppose a normal CP (i.e. $N_{\text {symb }}^{U L}$ equal to 7). Considering the allocated bandwidth $B=5 \mathrm{MHz}$, we obtain $N_{R B}=25$ available RBs to allocate to users at each TTI. The number of UEs per cell is denoted as $N_{U E}$. The metric used for our proposed heuristics is based on the effective Signal to Interference plus Noise Ratio $\left(\operatorname{SINR}_{e f f_{k}}\right)$ defined in [11]. It is the effective SINR experienced by each user $k$ over each RB. Using the mean instantaneous capacity method, the $\operatorname{SINR}_{e f f_{k}}$ is expressed as follows:

$$
\mathrm{SINR}_{\text {eff }}=2^{C_{k} / N_{s y m b}^{U L}}-1
$$

with $C_{k}$ the whole RB Shannon capacity computed as:

$$
C_{k}=\frac{1}{N_{s c}^{R B}} \cdot \sum_{i=1}^{N_{s y m b}^{U L}} \sum_{j=1}^{N_{s c}^{R B}} \log _{2}\left(1+\operatorname{SINR}_{k}^{(i, j)}\right)
$$

where $\operatorname{SINR}_{k}^{(i, j)}$ is the SINR experienced by user $k$ at each resource element $(i, j)$ for a given $\mathrm{RB}$ (with $1 \leq i \leq N_{\text {symb }}^{U L}$ and $1 \leq j \leq N_{s c}^{R B}$ ). The $\operatorname{SINR}_{k}^{(i, j)}$ can be computed using the following formula:

$$
\operatorname{SINR}_{k}^{(i, j)}=\frac{P_{k_{T x}}^{(i, j)} \cdot G_{t} \cdot G_{r}\left(\theta_{k}\right) \cdot \Lambda}{N+I^{(i, j)}}
$$

where $\Lambda$ is the total channel gain, expressed as:

$$
\Lambda=G_{c}\left(r_{k}\right) \cdot\left\|A_{f}^{(i, j)}\right\|^{2} \cdot A_{s}^{(i, j)}
$$

It includes $G_{c}\left(r_{k}\right)$ the path loss depending of $r_{k}$, the distance between the user $k$ and the BS. The random shadowing variations $A_{s}^{(i, j)}$ are assumed to be uncorrelated over the time-frequency grid and follow a log-normal distribution with parameter $\sigma_{s}$. However, we consider correlated variations of the time-frequency fading coefficients denoted $A_{f}^{(i, j)}$.

$P_{k_{T x}}^{(i, j)}$ is the transmission power of user $k$ over one resource element. Since the power is equally divided over all the resource elements of one RB, $P_{k_{T x}}^{(i, j)}$ can be expressed as:

$$
P_{k_{T x}}^{(i, j)}=\frac{P_{k_{T x}}}{N_{s y m b}^{U L} \cdot N_{s c}^{R B}}
$$

with $P_{k_{T x}}$ the RB mobile transmission power. $G_{t}$ is the mobile transmission antenna gain, $G_{r}$ is the BS antenna gain which depends on $\theta_{k}$, angle between UE $k$ and BS antenna boresight. $N$ is the thermal noise in the subcarrier and $I^{(i, j)}$ is the ICI level at each resource element $(i, j)$ obtained by Monte Carlo simulations. The details of the simulations parameters are given in section $\mathrm{V}$.

In this work, we consider an infinitely backlogged model in which, for each user, there is always data available for transmission. The scheduling is performed at each TTI. Thus, the BS allocates the $N_{R B}$ RBs every TTI. The throughput is averaged over 1000 TTI. The target BER is equal to $10^{-6}$. As the model respects the MCS robustness, the MCS selected for each user's transmission depends on the minimum $\operatorname{SINR}_{e f f_{k}}$ experienced by the concerned user on the whole allocated RBs. All users transmit at $P_{\text {max }}$, the maximum mobile transmission power set to $125 \mathrm{~mW}$, i.e. $21 \mathrm{dBm}$.

\section{Proposed RBs ALlocation Algorithms}

In a multi-user scenario, the users radio conditions may be different, considering their positions, velocity and environment. The channel dependent proposed RBs allocation algorithms consider these informations and use them as a metric when allocating RBs to users. Since the scheduling is performed each TTI [12], the metric used in this work is the mean effective SINR experienced by user $k$ at each RB $c$ over one TTI: $\overline{\operatorname{SINR}_{e f f_{k}}^{c}}$.

Let $\mathcal{K}$ be the set of users $\mathcal{K}=\left\{1, \cdots, k, \cdots, N_{U E}\right\}$ and $\mathcal{C}$ be the set of free RBs $\mathcal{C}=\left\{1, \cdots, c, \cdots, N_{R B}\right\}$. The RB allocation is considered as an optimization problem, where the desired solution is the mapping between users (i.e. set $\mathcal{K}$ ) and RBs (i.e. set $\mathcal{C}$ ) that maximizes the desired performance target. The optimal solution is provided by an exhaustive search as applied in branch-and-bound approach used in the mixed integer programming, but at the expense of an extraordinary complexity computation cost [1] [2]. In this section, an aggregate throughput maximization heuristic algorithm is proposed:

$$
\max \sum_{k=1}^{N_{U E}} R_{k}(t)
$$

where $R_{k}(t)$ is the total throughput of user $k$ at TTI $t$, over the whole allocated RBs, subject to:

1) the exclusivity of the allocated RBs: $\sum_{k=1}^{N_{U E}} x_{k}^{c}(t)=1 \quad \forall c \in \mathcal{C}$

2) the contiguity constraints:

$$
x_{k}^{j}(t)=0 \forall j>c+2 \text { if } x_{k}^{c}(t)=1 \text { and } x_{k}^{c+1}(t)=0
$$

3) the MCS robustness:

$$
R_{k}(t)=\min _{c \in A_{k}}\left(r_{k}^{c}(t)\right) .\left|\mathcal{A}_{k}\right|
$$

where, $\mathcal{A}_{k}$ is the set of RBs allocated to user $\mathrm{k}$ and $\left|\mathcal{A}_{k}\right|$ is its cardinal, $r_{k}^{c}(t)$ is the estimated instantaneous rate corresponding to the MCS and the effective SINR of UE $k$ in RB $c$ using an off-line look up table, and $x_{k}^{c}(t)$ denotes the RBs allocation for TTI $t: x_{k}^{c}(t)=1$ if the RB $c$ is allocated to user $k$, otherwise $x_{k}^{c}(t)=0$. 
The main steps of the allocation policy are summarized in Algorithms 1 and 2. The proposed heuristic algorithm first searches the pair (RB-UE) which maximizes the metric. Then it allocates the RB $c$ to the selected UE $k$ (Algorithm 1). To extend the allocation, the scheduler compares the metric values of user $k$ on the two adjacent RBs (left $c-1$ and right $c+1$ ) and selects the $\mathrm{RB} c^{\prime}$ corresponding to the highest metric value. The selected RB will be allocated to UE $k$ if (i) the allocation of this adjacent RB improves the throughput of UE $k$ and (ii) the maximum number of allocated RBs to the same user: $\alpha_{k_{\max }}$, is not reached for UE $k$, as detailed in Algorithm 2. We propose two heuristics: 1) OEA: an opportunistic RB allocation which allows users to have all RBs (i.e. $\alpha_{k_{\max }}=N_{R B}$ ), and 2) QoS based OEA: a less opportunist algorithm which adapts the set of assigned RBs to the desired QoS, with $\alpha_{k_{\max }}$ determined as follows :

$$
\alpha_{k_{\text {max }}}=\left\lceil\frac{\mathrm{SINR}_{\text {target }}}{\min \left(\overline{\mathrm{SINR}_{\text {eff }}^{c}}\right)}\right\rceil \forall c \in \mathcal{C}
$$

Due to the transmission power update of user $k$ over each RB, an update of the metric $\left(\overline{\operatorname{SINR}_{e f f_{k}}^{c}}\right)$ is performed before each expansion using the following formula:

$$
\overline{\operatorname{SINR}_{e f f_{k}}^{c}}=\overline{\operatorname{SINR}_{\text {eff } f_{k}}^{c}}-10 \log \left(\left|\mathcal{A}_{k}\right|+1\right) \quad \forall c \in \mathcal{C}
$$

The output of both algorithms OEA and QoS based OEA is $\mathcal{S}_{R B}$ : the set of allocated (RB-UE) pairs.

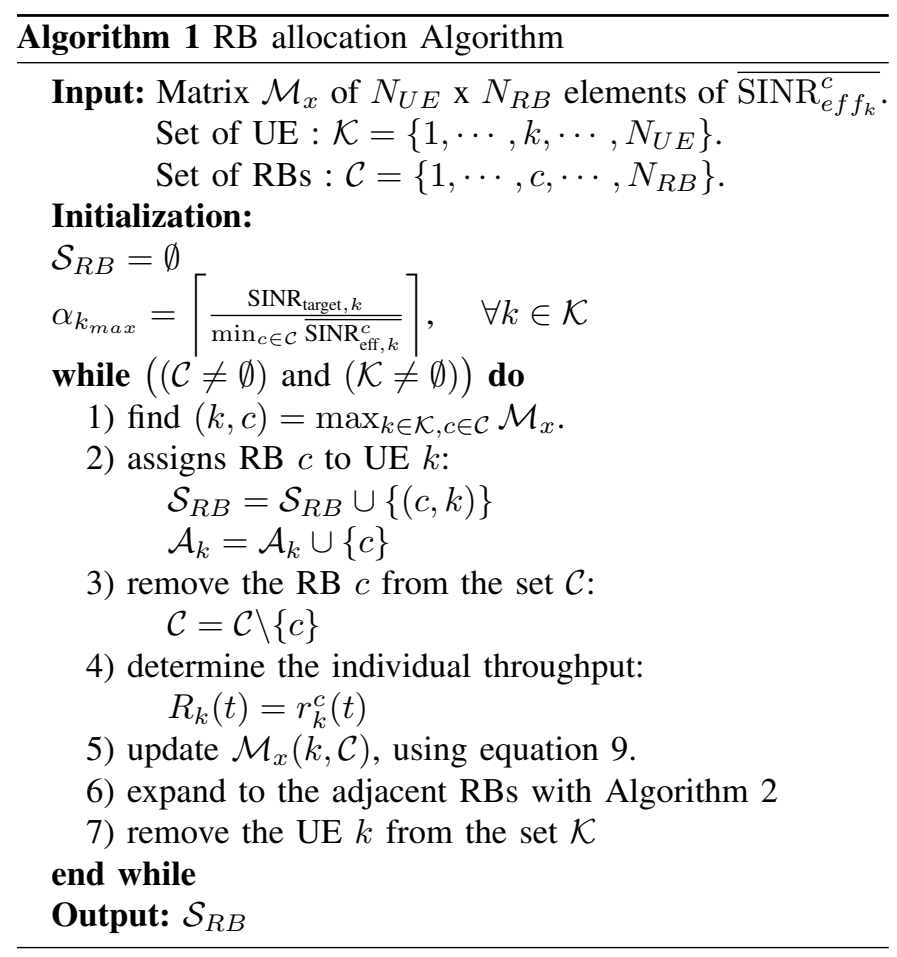

\section{COMPLEXITY COMPUTATION}

In this section, we compute and compare the computational complexity of the proposed algorithms with that of reference algorithms in literature: FDPS-LMF, HLGA and RME.

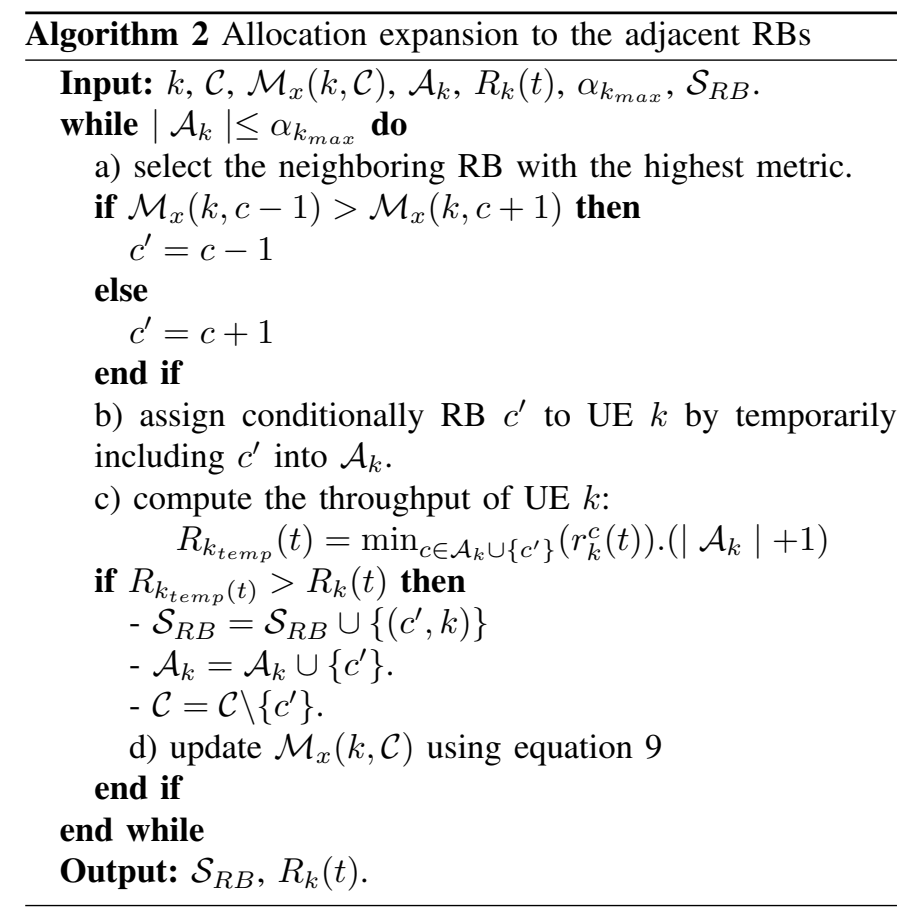

\section{A. OEA and QoS based OEA complexity}

Step 1) of Algorithm 1 is performed after a linear search on the remaining $\mathrm{RBs}$ and users in sets $\mathcal{C}$ and $\mathcal{K}$. At the worst case, each user will be assigned only one RB. Then, the computational complexity of the $l^{\text {th }}$ run of this step is upper-bounded by $\mathcal{O}\left(\left(N_{U E}-l\right)\left(N_{R B}-l\right)\right)$. The throughput computation is performed with respect to the MCS robustness (equation 7) by looking for the minimum rate in the set $\mathcal{A}_{k}$. Hence, the computational complexity of steps 4) in Algorithm 1 and step c) in Algorithm 2 is $\mathcal{O}\left(N_{R B}-l\right)$. The user's metric update (steps 5) and d)) is performed before each new RB allocation at the expense of a computational complexity of $\mathcal{O}\left(N_{R B}-l\right)$. The RBs allocation expansion must be done as long as $\alpha_{k_{\max }}$ is not reached. This parameter $\alpha_{k_{\max }}$ depends on the QoS desired by each user and is usually quite low for QoS based OEA. Then, at the worst case represented with OEA $\left(\alpha_{k_{\max }}=N_{R B}\right)$, step 6) will be run $\mathcal{O}\left(N_{R B}-l\right)$ times. Both algorithms stop allocating the RBs when all the users are served or when there is no more RB to be allocated. Therefore, $l$ is upper-bounded by $\min \left(N_{R B}, N_{U E}\right)$ and the expression of the proposed algorithms OEA and QoS based OEA complexity $\mathcal{C}_{O}$ is:

$$
\begin{array}{r}
\mathcal{C}_{O}=\sum_{l=0}^{\min \left(N_{R B}, N_{U E}\right)}\left[\mathcal{O}\left(\left(N_{U E}-l\right) \cdot\left(N_{R B}-l\right)\right)\right. \\
\left.+\mathcal{O}\left(N_{R B}-l\right)+2 . \mathcal{O}\left(\left(N_{R B}-l\right)^{2}\right)\right]
\end{array}
$$

After some mathematical derivations, we obtain the following complexity:

$$
\begin{gathered}
\mathcal{C}_{O_{N_{U E} \geq N_{R B}}}=\mathcal{O}\left(\frac{N_{R B}^{3}}{2}+\left(\frac{N_{U E}}{2}-\frac{1}{2}\right) N_{R B}^{2}-\frac{N_{U E}}{2} N_{R B}\right) \\
\mathcal{C}_{O_{N_{U E} \leq N_{R B}}}=\mathcal{O}\left(\frac{N_{U E}^{3}}{2}+\left(\frac{1}{2}-\frac{3 . N_{R B}}{2}\right) N_{U E}^{2}\right)+ \\
\mathcal{O}\left(\left(2 N_{R B}^{2}-\frac{3 N_{R B}}{2}\right) N_{U E}\right)
\end{gathered}
$$


Thus, the computational complexity of OEA and QoS based OEA is polynomial of order 3 .

\section{B. FDPS-LMF and HLGA complexity}

FDPS-LMF and HLGA algorithms both perform a linear search on RBs and users to find the pairs (RB-UE). This step can be done using sorted metrics values and saving the corresponding pairs (RB-UE). The computational complexity of the metrics values sorting is $\mathcal{O}\left(N_{R B}^{2} N_{U E}^{2}\right)$ in the worst case. Using the quick sort algorithm [13], the complexity is reduced to $\mathcal{O}\left(N_{R B} N_{U E} \cdot \log \left(N_{R B} N_{U E}\right)\right)$. Then, both algorithms allocate the RBs to the corresponding users considering the contiguity constraint. The complexity for these steps is $\mathcal{O}\left(N_{U E} N_{R B}-2\right)$. The FDPS-LMF stops the RBs allocation at this step, whereas the HLGA allocates the free remaining $\mathrm{RBs}$ at the expense of $\mathcal{O}\left(\left(N_{R B}-1\right) \cdot\left(N_{U E}-1\right)\right)$ additional operations. Therefore the final complexity for the FDPS-LMF: $\mathcal{C}_{F}$, and the HLGA: $\mathcal{C}_{H}$, are respectively:

$\mathcal{C}_{F}=\mathcal{O}\left(N_{R B} N_{U E} \cdot \log \left(N_{R B} N_{U E}\right)\right)+\mathcal{O}\left(N_{U E} \cdot\left(N_{R B}-2\right)\right)$

and

$$
\begin{aligned}
\mathcal{C}_{H} & =\mathcal{O}\left(N_{R B} N_{U E} \cdot \log \left(N_{R B} N_{U E}\right)\right)+ \\
& \mathcal{O}\left(N_{U E} \cdot\left(N_{R B}-2\right)\right)+\mathcal{O}\left(\left(N_{U E}-1\right) \cdot\left(N_{R B}-1\right)\right)
\end{aligned}
$$

\section{RME complexity}

The RME algorithm also performs the linear search of the first pair (RB-UE). In fact the complexity for the first step is $\mathcal{O}\left(\left(N_{R B}-l\right)\left(N_{U E}-l\right)\right)$. The complexity of the RME expansion step that recursively finds the users adjacent RB highest metric value is in $\mathcal{O}\left(\left(N_{R B}-1\right) .\left(N_{U E}-1\right)\right)$. The allocation is done till all users are served or there is no more remaining free $\mathrm{RB}$. The same management method for these remaining free RBs as in HLGA is used. Therefore the computational complexity evaluation of the RME: $\mathcal{C}_{R}$, is :

$$
\begin{aligned}
\mathcal{C}_{R}=\sum_{l=0}^{\min \left(N_{R B}, N_{U E}\right)} & {\left[\mathcal{O}\left(2\left(N_{U E}-l\right)\left(N_{R B}-l\right)\right)\right] } \\
+ & \mathcal{O}\left(\left(N_{R B}-1\right) .\left(N_{U E}-1\right)\right)
\end{aligned}
$$

which can be also expressed as follows:

$\mathcal{C}_{R_{N_{U E} \geq N_{R B}}}=\mathcal{O}\left(\frac{-N_{R B}^{3}}{3}+N_{U E} N_{R B}^{2}-\frac{2 N_{R B}}{3}+\left(1-N_{U E}\right)\right)$

$\mathcal{C}_{R_{N_{U E} \leq N_{R B}}}=\mathcal{O}\left(\frac{-N_{U E}^{3}}{3}+N_{R B} N_{U E}^{2}-\frac{2 N_{U E}}{3}+\left(1-N_{R B}\right)\right)$

\section{Comparison of the algorithms complexity}

All the algorithms studied in this paper have a polynomial complexity that depends on $N_{R B}$ and $N_{U E} \cdot N_{R B}$ is determined by the system bandwidth and is set to $N_{R B}=25$. Figure 1 represents the ratio between the computational complexity of the algorithms: FDPS-LMF, HLGA and RME, and the complexity of OEA and QoS based OEA, depending on the number of users $N_{U E}$. Note that the FDPS-LMF is the least complex scheduler, thanks to the quick sort algorithm. The gap between FDPS and HLGA is caused by the remaining free RBs management. Although our OEA algorithms update the metrics before each RB allocation, their complexity is lower than the RME one when $N_{U E}$ exceeds $N_{R B}\left(N_{U E} \geq 40\right)$.

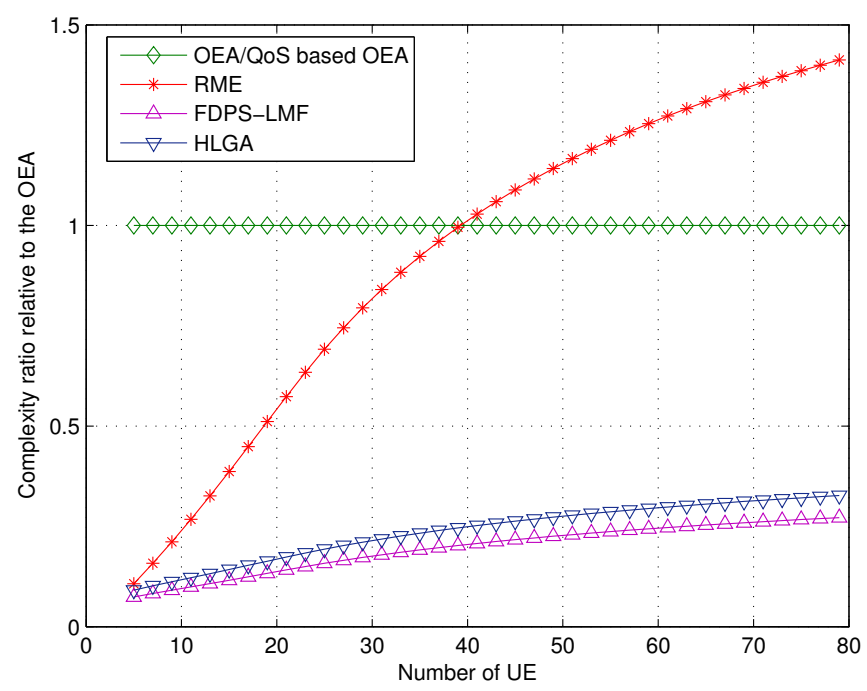

Fig. 1. Computational complexity of the studied algorithms with $N_{R B}=25$

\section{Performance eValuation}

\section{A. Simulation parameters}

To evaluate the proposed algorithms performances, we compare OEA and QoS based OEA with: 1) three proportional fair schedulers cited before (RME, FDPS-LMF and HLGA) and 2) one Opportunistic RB allocation algorithm that uses the same allocation policy as RME but differs from it by considering the radio channel conditions of each user (i.e. $\operatorname{SINR}_{e f f_{k}}$ ) as a metric (with no update after each RB allocation).

The simulation parameters are summarized in table I. We consider the RB allocation in the central cell where users are uniformly distributed. One QoS class is considered, with a target throughput of $1 \mathrm{Mbps}$ for QoS based OEA.

\begin{tabular}{|l|l|}
\hline Cellular layout & Hexagonal grid,19 tri-sector cells. \\
\hline Max/ Min UE-BS distance & $500 \mathrm{~m} / 30 \mathrm{~m}$ \\
\hline Carrier frequency & $2.6 \mathrm{GHz}$ \\
\hline System bandwidth & $B=5 \mathrm{MHz}$ per sector $\Rightarrow N_{R B}=25$ \\
\hline Duration & $1000 \mathrm{TTI}$ \\
\hline User power class & $P_{\max }=21 \mathrm{dBm}(125 \mathrm{~mW})$ \\
\hline Radio channel gain & Okumura Hata for urban areas: \\
& $G_{c}\left(r_{j c}\right)=10^{-a} / 10 * r_{j c}^{-b / 10}$. \\
& $a=136.7$ and $b=34.4$. \\
\hline BS antenna pattern & $G_{r}\left(\theta_{j c}\right)=-\min \left[12 *\left(\frac{\theta_{j c}}{\theta_{3 d B}}\right)^{2}, \beta\right]$. \\
& $\theta_{3 d B}=70^{\circ}, \beta=20 \mathrm{~dB}$ \\
\hline User antenna gain & $G_{t}=0 \mathrm{dBi}$ \\
\hline Rayleigh fading & coef corr $=0.5, \mathrm{UE}$ velocity $=3 \mathrm{~km} / \mathrm{h}$ \\
\hline Log-normal shadowing & $\sigma_{s}=5 \mathrm{~dB}$ \\
\hline MCS setting & QPSK $1 / 2,2 / 3,3 / 4$ \\
& 16 QAM $1 / 2,2 / 3,3 / 4$ \\
& 64 QAM $1 / 2,2 / 3,3 / 4$ \\
\hline
\end{tabular}

TABLE I

SIMULATION PARAMETERS 


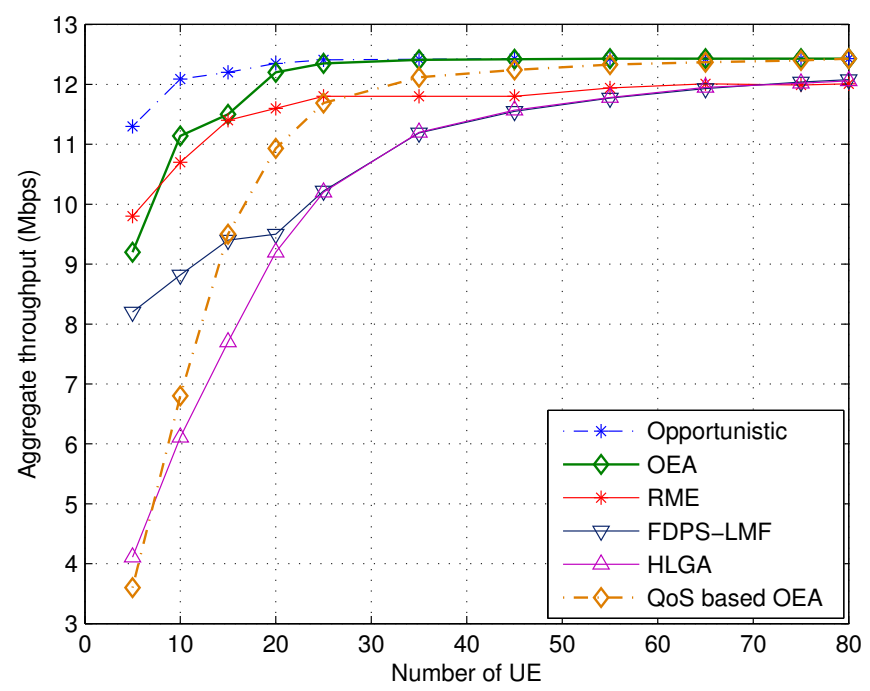

Fig. 2. Aggregate throughput with $N_{R B}=25$

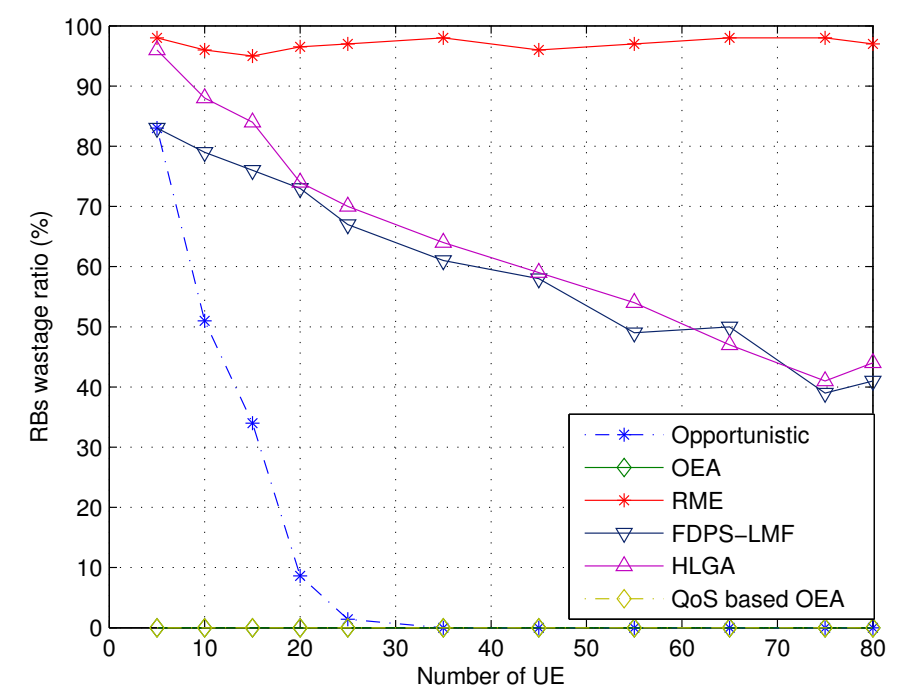

Fig. 3. RBs wastage Ratio

\section{B. Numerical results}

The satisfaction level system requirement is the maximization of the aggregate throughput. Figure 2 shows the aggregate throughput of the central cell obtained with the studied algorithms. The aggregate throughput increases when the load of the cell increases. The OEA aggregate throughput is higher than the aggregate throughput of the proportional fair schedulers (RME, FDPS-LMF and HLGA) and close to the Opportunistic one. The aggregate throughput generated by QoS based OEA increases significantly to be higher than the proportional fair schedulers when $N_{U E} \geq N_{R B}$. At low load, the free RBs management applied by the HLGA decreases the aggregate throughput level compared to FDPS.

When the number of UEs exceeds the number of RBs, OEA and QoS based OEA offer good performances compared to the RME algorithm. Actually, they achieve a higher aggregate throughput with less computational complexity than RME algorithm. Unlike RME, FDPS-LMF and HLGA algorithms are less complex than proposed algorithms. However, they achieve a significantly lower aggregate throughput; the gap with OEA varie from more than $200 \%$ at low load, to $20 \%$ when $N_{U E}=N R B$. It then decreases but is still around 5\% when $N_{U E} \geq 2 . N_{R B}$. Then, a trade-off between complexity and throughput would suggest to use our algorithms.

To enlighten the metrics update benefits, we compute the maximum RB wastage ratio of each algorithm over the simulation duration. The RB wastage ratio is the ratio between the number of RBs allocated to UE having a zero throughput and the total number of RBs. Figure 3 shows that OEA and QoS based OEA completely cancel this wastefulness. The RB wastage ratio of the Opportunistic scheduler decreases to cancel when $N_{U E} \geq N_{R B}$, but the RME wastes around $98 \%$ of RBs whatever $N_{U E}$, due to the proportional fair metric. It means that up to 98 RBs out of 100 are allocated to users but do not lead to any data transmission. Considering FDPS-LMF and HLGA algorithms, the RB wastage ratio tend to $40 \%$, as they compensate the proportional fair metric by their allocation policy. In addition to the high aggregate throughput and the cancelation of RB wastefulness, the OEA efficiency resides in the number of served users. Actually, the user throughput improvement constraint associated to the MCS robustness limits the number of RBs allocated to each user, hence giving a chance to other users to be served. Figure 4 represents the ratio of unallocated RBs. At low load the OEA efficiently uses $10 \%$ of the available resources. The ratio of unallocated RBs decreases and is cancelled when $N_{U E}$ increases, under appropriate user radio conditions.

\section{CONCLUSION}

In this paper we investigated the RB allocation in uplink LTE networks. We presented the constraints that a scheduler must consider in the uplink due to the SC-FDMA technique. We proposed two algorithms: OEA and QoS based OEA, for which we computed analytically the complexity and compared the performances to proportional fair and opportunistic RB allocations. Most of the time, OEA offers higher aggregate throughput than RME, and is close to the maximum one, even though it has a lower polynomial complexity. In addition, the metric updates provided by our proposed heuristics cancel the radio capacity wastage. The QoS based OEA allows to adapt the RB allocation with the desired QoS. At low load, the remaining free RBs offer the possibility to use our algorithms in coordinated Multipoint (CoMP) networks. In a future work, we will study the performances of our RB allocation heuristics in a CoMP network with different QoS classes, applying power control to the mobile transmission power. 


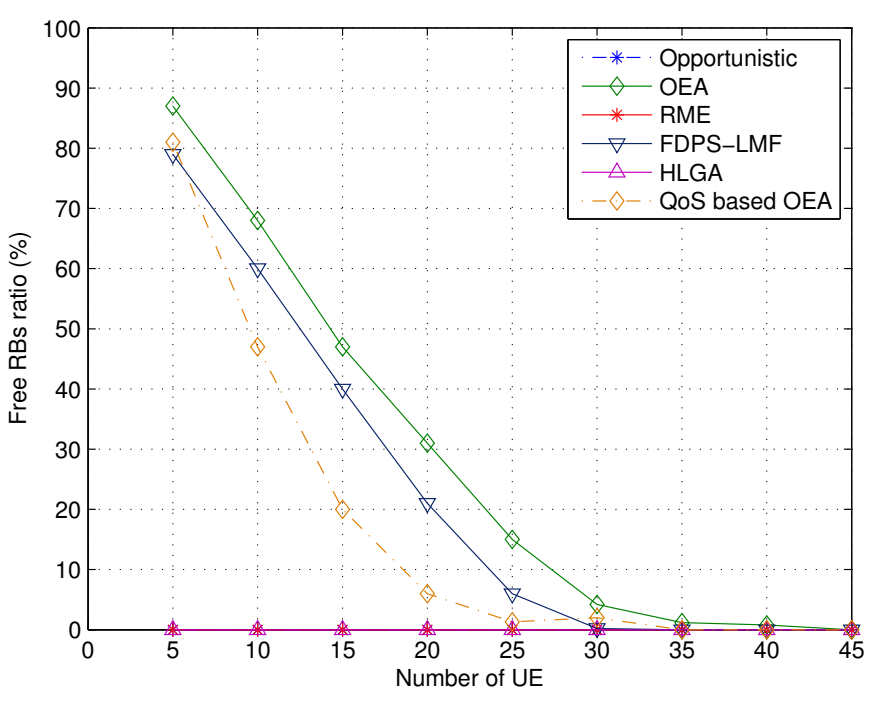

Fig. 4. Free RBs ratio

\section{REFERENCES}

[1] I. C. Wong, O. Oteri, and W. McCoy, "Optimal resource allocation in uplink sc-fdma systems," Wireless Communication, IEEE Transactions on, vol. 8, pp. 2161-2165, May 2009.
[2] F. I. Sokmen and T. Girici, "Uplink resource allocation algorithms for single-carrier fdma systems," in European Wireless Conference, Lucca, Tuscany, Italy, April 2010.

[3] O. Nwamadi, X. Zhu, and A. Nandi, "Dynamic subcarrier allocation for single carrier-fdma systems," in EUSIPCO, Lausanne, Switzerland, August 2008

[4] J.-H. Noh and S.-J. Oh, "Distributed sc-fdma resource allocation algorithm based on the hungarian method," in Vehicular Technology Conference Fall, Anchorage, Alaska, USA, September 2009.

[5] M. Al-Rawi, R. Jantti, J. Torsner, and M. S. gfors, "Opportunistic Uplink Scheduling for 3G LTE Systems," in Innovations in Information Technology, Dubai, UEA, November 2007.

[6] S.-B. Lee, L. Pefkianakis, A. Meyerson, S. Xu, and S. Lu, "Proportional Fair Frequency-Domain Packet Scheduling for 3GPP LTE Uplink," in INFOCOM, April 2009, pp. 2611 - 2615.

[7] L. Á. M. R. de Temiño, G. Berardinelli, S. Frattasi, and P. E. Mogensen, "Channel-aware scheduling algorithms for sc-fdma in lte uplink," in PIMRC, September 2008.

[8] A. L. Stolyar, "On the asymptotic optimality of the gradient scheduling algorithm for multiuser throughput allocation," Operations Research, vol. 53, no. 1, pp. 12-25, 2005.

[9] H. Holma and A. Toskala, LTE for UMTS: Evolution to LTE-Advanced. Wiley, 2010.

[10] Evolved Universal Terrestrial Radio Access (E-UTRA); Physical channels and modulation, 3GPP TS 36.211, V8.6.0 Std., March 2009.

[11] K. Ramadas and R. Jain, "Wimax system evaluation methodology," Technical report, January 2007.

[12] Evolved Universal Terrestrial Radio Access (E-UTRA); User Equipment (UE) radio transmission and reception, 3GPP TS 36.101 , V10.3.0 Std., June 2011.

[13] R. Sedgewick and P. Flajolet, An introduction to the analysis of algorithms. Addison-Wesley-Longman, 1996. 